\title{
CBProf: Customisable Blockchain-as-a-Service Performance Profiler in Cloud Environments
}

\author{
Ruyue Xin, Jardenna Mohazzab, Zeshun Shi, and Zhiming Zhao \\ Multiscale Networked Systems (MNS) research group, University of Amsterdam, \\ Amsterdam, Netherlands \\ $\{r . x i n \mid$ z.shi2 $\mid$ z.zhao $\}$ @uva.nl, jardenna.nl@gmail.com
}

\begin{abstract}
Blockchain technologies, e.g., Hyperledger Fabric and Sawtooth, have been evolving rapidly during past years and enable potential decentralised innovations in a substantial amount of business applications, e.g. crowd journalism, car-sharing and energy trading. The development of decentralised business applications has to face challenges in selecting suitable blockchain technologies, customising network protocols among distributed peers, and optimising system performance to meet application requirements. Also, manually testing and comparing those different technologies are time-consuming. Therefore, an effective tool is needed for profiling the performance characteristics of blockchain services in different cloud environments. In this paper, we present the Customisable Blockchain-as-a-Service Performance Profiler (CBProf), a tool we developed for automating blockchain deployment and performance profiling in cloud environments. We also provide the implementation and functionality demonstration of this tool.
\end{abstract}

Keywords: Blockchain - Blockchain-as-a-Service $($ BaaS $) \cdot$ Automatic deployment $\cdot$ Performance profiling

\section{Introduction}

Blockchain technologies enable a secure, transparent, and decentralised environment to support transactions of data, goods, or financial resources [1]. Those technologies bring lots of opportunities for enterprises to enhancing their business processes in a decentralised manner. Permissioned blockchains are typical examples; they allow trusted and authorised entities to engage in blockchain activities to ensure privacy and security of enterprise information [13]. Cloud environments provide elastic and on-demand resources for customising data storage, processing, and communication, which play an increasingly important role for enterprises to operate blockchain-based decentralised applications (DApps) [12,15].

Permssioned blockchains like Hyperledger Fabric and Sawtooth have developed in recent years, and cloud resource is facilitated by an increasing number of providers, such as Amazon Web Services (AWS) and Microsoft Azure [5] [4]. Therefore, developers need to ascertain the blockchain technologies and cloud provides best suited for their business purpose when transitioning from a classical 
application to a DApp [10]. To customise the configuration of a DApp in cloud environments, it is essential to determine the performance characteristics of different blockchains in different cloud situations, while profiling the performance of blockchains manual is rather time-consuming and undesirable [11]. Therefore, it becomes an urgent need to create a user-friendly tool to automatically deploy different configurations of blockchains on demand and provide insight into the performance by benchmarking the performance profiling results.

This paper presents a tool called Customisable Blockchain-as-a-Service Performance Profiler (CBProf), which implements automatic blockchain deployment on cloud environments and performance benchmarking and profiling for various blockchains. The rest of the paper is organised as follows: Section 2 analyses the requirements and describes related works. Section 3 presents CBProf architecture and function of each component. Section 4 is the implementation and demonstration of CBProf. Finally, section 5 concludes this paper and provides a discussion about future work.

\section{Requirements and Related Works}

In this section, we analyse the requirements and challenges of a user-friendly blockchain performance profiler and provide related works based on requirements.

\subsection{Requirements and Challenges}

A user-friendly performance profiling tool is needed for developing and operating DApps in cloud environments. The tool should support a user to effectively customize the configuration of a blockchain network, automate the deployment of the blockchain services in cloud infrastructures, and explore the performance information collected from the runtime system. The tool has to consider the scenarios where the blockchain application has to run across different providers, e.g. for the reasons of improving fault tolerance, security and performance. However, the deployment of blockchain services across a distributed cloud environment involves provisioning of virtual machines (VMs), installing blockchain services, and customising the connectivity among blockchain nodes, which is not simple. Moreover, the performance of blockchains is influenced by many factors in the distributed cloud environment [7]; it is a challenge to build a precise performance model for a decentralized application.

\section{$2.2 \quad$ Related Works}

Based on the requirements of the user-friendly tool, we identify related works with automatic blockchain deployment and blockchain performance profiling.

Automatic blockchain deployment. Blockchain deployment on distributed environments is typically a time-consuming and cumbersome process, which leads to the research about automatic blockchain deployment. Frantz et al. 
propose a modelling approach that supports the semi-automated translation of human-readable contract representations into computational equivalents [2]. However, the automation in this research is only about smart contracts. Research by Shi et al. proposes a framework to automate the provision of required infrastructures and deploy Hyperledger Sawtooth [8]. We extend the research in this paper and provide a tool that supports more blockchain and is more user-friendly.

Automatic deployment tools such as Puppet ${ }^{1}$ and Ansible ${ }^{2}$ are commonly used but hard to integrate into invocation level automation, which is crucial for a user-friendly automatic deployment tool. However, CloudsStorm [14] which is developed for automatic deployment of cloud applications, can leverage different clouds and program them into cloud applications. Therefore, we develop our blockchain deployment tool integrating CloudsStorm. This tool is also integrated into our toolkit Software Defined Infrastructure Automator (SDIA) ${ }^{3}$.

Blockchain performance profiling. For DApps, performance is a critical factor to consider according to application requirements. There already are many studies that focus on blockchain performance profiling. Pongnumkul et al. have contributed to performance analysis of Ethereum and Hyperledger Fabric with a varying number of transactions [6]. Shi et al. have studied the performance of one of Hyperledger Sawtooth in cloud environments [7]. The results provide insights for blockchain operators to optimise the performance of Sawtooth by adjusting configuration parameters. Currently, blockchain performance studies usually focus on several metrics or specific platforms $[3,6,9]$. Therefore, these studies cannot be used as a baseline when deploying a blockchain.

From related works, we can see that research into automation of blockchain deployment and performance profiling is very recent and usually separated into two topics. Therefore, an advanced framework is required to automate the complete process of blockchain deployment and performance profiling.

\section{Customisable Blockchain-as-a-Service Performance Profiler}

To meet the requirements in section 2, we provide a performance profiler called CBProf in this section. We will introduce the functionality of each component in CBProf and how it works in detail.

\subsection{Architecture and Functional Components}

In CBProf (seen in figure 1), we design a blockchain deployment tool for automatic blockchain deployment and benchmarking and profiling tool for testing and profiling blockchain performance. Each tool contains several components.

\footnotetext{
1 https://puppet.com/

${ }^{2}$ https://www.ansible.com/

${ }^{3}$ https://github.com/QCDIS/sdia-deployer
} 
Blockchain deployment tool. This tool processes blockchain deployment requests and facilitates deployment automatically. In figure 1 , the user input retriever component collects blockchain deployment requests from users at first, such as VMs type, number and provider. After receiving blockchain configuration requirements, CloudsStorm, which is a framework for managing an applicationdefined infrastructure, is used for automatic deployment.

When deploying a blockchain in clouds, the nodes of a blockchain network are represented by VMs in clouds, and the nodes must form a network to ensure their communication with each other. Therefore, the blockchain deployment tool includes functions of VMs creation and communication. As for customised blockchain deployment, the blockchain deployment tool supports different blockchain platforms, such as Hyperledger Fabric and Sawtooth, and on different cloud provides, such as AWS and ExoGeni. All the blockchain services will be started automatically after deployment.

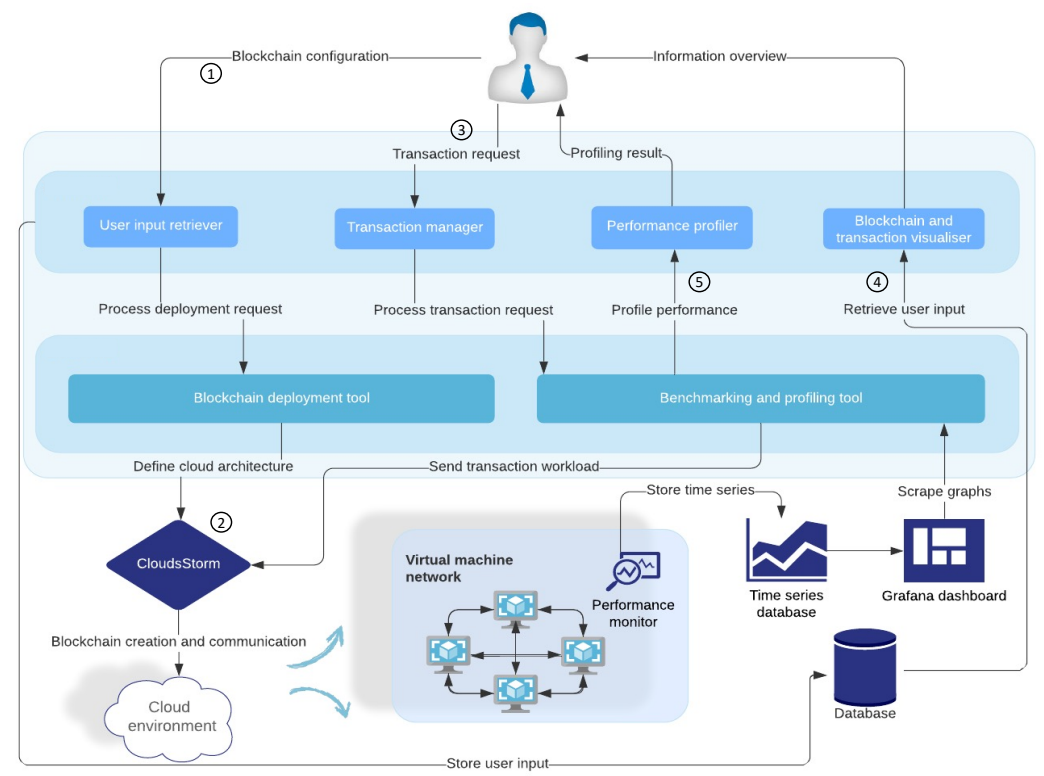

Fig. 1. Architecture of CBProf including blockchain deployment tool and benchmarking and profiling tool.

Benchmarking and profiling tool. The benchmarking tool processes and formats transaction requests so that it can simulate different business scenarios through which the functionality of the blockchain can be benchmarked. The profiling tool specifies the deployment of a performance monitor that records all blockchain processes, and it allows the monitor to collect the real-time per- 
formance data and stores it in a time-series database. Also, it can be used for profiling blockchain performance.

In figure 1, the transaction manager component allows for the specification, retrieval and storage of customised transaction requests, such as execution time and send rate of workloads can be ordered. Benchmark tools can be integrated into this component. After benchmarking, the performance profiler provides an overview and comparison of blockchain performance, such as the transaction latency and throughput (TPS). The comparison can provide users with a clear perspective regarding a specific purpose or a certain performance measure. As for blockchain and transaction visualiser, we design this component for collecting and visualising real-time blockchain performance data. The time-series database provides persistent storage on the local file system. And, a dashboard is configured to visualise data collected by the database. With this component, we can check many performance metrics like block number, committed transactions in real-time. This component is also interactive so that users can customise the performance of a specific period.

\subsection{How CBProf Works}

CBProf is a customisable automatic blockchain deployment and benchmarking tool. About customisation, CBProf provides choices with a) blockchain configuration including cloud provider, data centre, VMs number(blockchain nodes number), VMs type, blockchain platforms, and b) transaction request including execution time and send rate.

As shown in figure 1, CBProf works follow the process: 1) blockchain configuration is sent from users and stored in a database. 2) CloudsStorm will start the automatic deployment, including VMs launching, network configuration and VMs initialisation. Also, with CloudsStorm, blockchain initialisation on created VMs will be done at the same time. 3) After the blockchain is configured, transaction requests can be processes which is blockchain benchmarking with different patterns of transactions. 4) With a blockchain and transaction visualiser, users can check real-time blockchain performance. 5) Through a performance profiler, users can compare blockchain performance with different blockchain or transaction configurations. With CBProf, quick insight into the general performance of a blockchain can be acquired. Alternatively, when more in-depth and personalised analysis is desired, customised benchmark experiments can be configured by specifying benchmark artefacts.

\section{Implementation and Demonstration}

This section provides the implementation of CBProf and gives profiling results of blockchain performance comparing different configurations. 


\subsection{Implementation}

We implement CBProf with many technologies. For the blockchain deployment tool, customised blockchain configuration is implemented by defining sub-topology, top-topology, infrastructure and execution code in CloudsStorm. Here, a script that accepts deployment configurations and automatically writes the code to create the blockchain configuration is also provided. In benchmarking and profiling tool, we integrate Hyperledger Caliper ${ }^{4}$ for benchmarking the performance of blockchains. And, we deploy Prometheus ${ }^{5}$ and Grafana ${ }^{6}$ to collect and visualise real-time blockchain performance data. Besides, we use $\mathrm{MongoDB}^{7}$ as the database to store user input information, and we build the GUI (graphical user interface ) with Vue.js 8 .

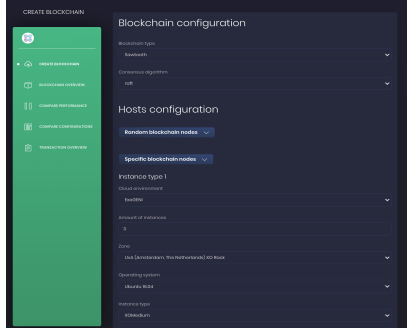

(a)

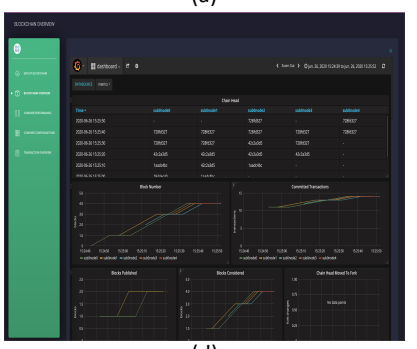

(d)

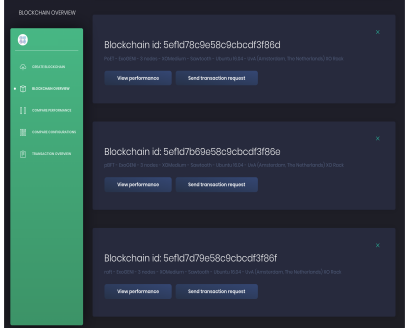

(b)

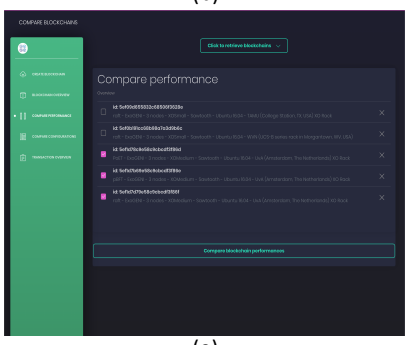

(e)

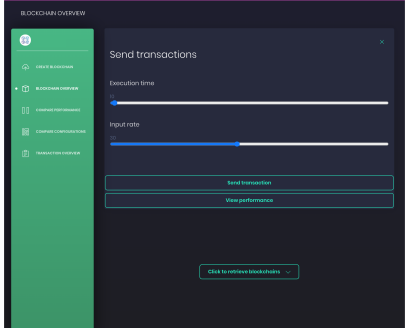

(c)

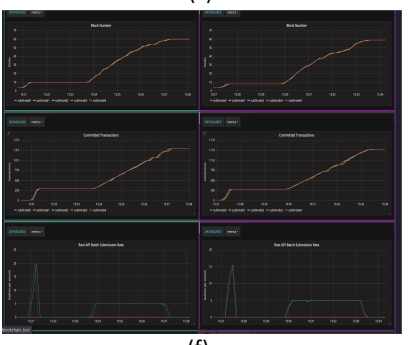

(f)

Fig. 2. CBProf tool interface and functions: (a) customise blockchain configuration; (b) overview of deployed blockchains; (c) transaction request; (d) performance visualization; (e) compare blockchain configuration; (f) compare blockchain performance

\subsection{Functionality Demonstrate}

The tool interface can be seen in figure 2. The interface includes five functions: creating a blockchain, blockchain overview, comparing performance, comparing

\footnotetext{
${ }^{4}$ https://github.com/hyperledger/caliper

${ }^{5}$ https://prometheus.io/

6 https://grafana.com/

${ }^{7}$ https://www.mongodb.com/

8 https://vuejs.org/
} 
configuration and blockchain overview, which correspond to the architecture and components in section 3.1. Users can use it to deploy different blockchains such as Hyperledger Fabric or Sawtooth on different cloud environments. Also, users can benchmark a blockchain with different transaction requests. Performance metrics can be seen in real-time during blockchain execution. And users can compare the performance of multiple blockchain configurations.

We also test the automatic deployment of Hyperledger Fabric on ExoGeni and AWS with CBProf. At first, for automatic blockchain deployment, we set up different fabric networks with 3,6,9,12,15 nodes and deploy them on ExoGeni and AWS clouds, respectively. All organisations of fabric network are a member of a single channel. VMs of ExoGeni are all XOSmall, and VMs of AWS are all t2.Small. Figure 3 shows the execution overhead of automatic deployment. It's obvious that ExoGeni consumes more time than AWS for any node number. Also, we can see that the consumption time of different nodes is similar, which means that the deployment is synchronised, and large-scale automatic blockchain deployment is possible with CBProf.

As for performance profiling, we provide a comparing of blockchain performance with different transaction requests. We do the benchmark for a three nodes fabric network. The transaction requests include both read and write operations. We set up send rates as 10, 20, 30, 40, 50 tps (transactions per second), and transaction duration as 100s. Performance profile results can be seen in figure 4. Throughput is higher for query operation comparing with init operation, which is easy to understand because read operation needs fewer resources. And then, we can see that the throughout gradually stabilises when send rate is higher than 40. Therefore, send rate of 40 may be a bottleneck of this blockchain configuration. Performance profiling gives us an insight into blockchains capacity.
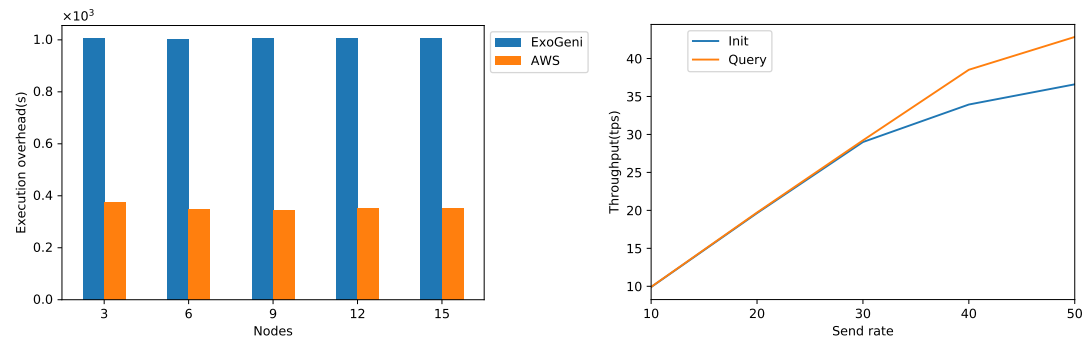

Fig. 3. Provision time of blockchain de- Fig. 4. Throughput of blockchain perforployment mance

\section{Conclusion}

In this paper, we provide the design and implementation of Customisable Blockchainas-a-Service Performance Profiler (CBProf), a user-friendly tool that allows for 
convenient blockchain deployment and performance profiling. CBProf provides an approach to build a generic functionality that automatically defines the cloud architecture, the virtual machine network and the execution code to deploy the blockchain. Also, CBProf provides an approach to monitor the blockchain processes, visualise transaction execution processes and compare blockchain performance. Further in this paper, we present the implementation and functionality demonstration of CBProf. Moreover, we test the automation deployment for Hyperledger Fabric on cloud environments and provide the performance profiling results under different transaction requests. The results give us an overview of blockchain capacity under specific cloud environments.

The CBProf also collects many other monitor metrics such as resource usage and transaction flow data. In the future, we will develop more methods to detect anomalies for blockchain applications and provide adaptation strategies for meeting users requirements. Our tool allows for extension with open-source machine learning methods. We aim to make it an open framework and attract community effort to support more DApps related technologies.

\section{ACKNOWLEDGMENT}

This research is funded by the EU Horizon 2020 research and innovation program under grant agreements 825134 (ARTICONF project), 862409 (BlueCloud project) and 824068 (ENVRIFAIR project).

\section{References}

1. Abeyratne, S.A., Monfared, R.P.: Blockchain ready manufacturing supply chain using distributed ledger. International Journal of Research in Engineering and Technology 5(9), 1-10 (2016)

2. Frantz, C.K., Nowostawski, M.: From institutions to code: Towards automated generation of smart contracts. In: 2016 IEEE 1st International Workshops on Foundations and Applications of Self* Systems (FAS* W). pp. 210-215. IEEE (2016)

3. Hao, Y., Li, Y., Dong, X., Fang, L., Chen, P.: Performance analysis of consensus algorithm in private blockchain. In: 2018 IEEE Intelligent Vehicles Symposium (IV). pp. 280-285. IEEE (2018)

4. Kotas, C., Naughton, T., Imam, N.: A comparison of amazon web services and microsoft azure cloud platforms for high performance computing. In: 2018 IEEE International Conference on Consumer Electronics (ICCE). pp. 1-4. IEEE (2018)

5. Liu, B., Yu, X.L., Chen, S., Xu, X., Zhu, L.: Blockchain based data integrity service framework for iot data. In: 2017 IEEE International Conference on Web Services (ICWS). pp. 468-475. IEEE (2017)

6. Pongnumkul, S., Siripanpornchana, C., Thajchayapong, S.: Performance analysis of private blockchain platforms in varying workloads. In: 2017 26th International Conference on Computer Communication and Networks (ICCCN). pp. 1-6. IEEE (2017)

7. Shi, Z., Zhou, H., Hu, Y., Jayachander, S., de Laat, C., Zhao, Z.: Operating permissioned blockchain in clouds: A performance study of hyperledger sawtooth. In: 2019 18th International Symposium on Parallel and Distributed Computing (ISPDC). pp. 50-57. IEEE (2019) 
8. Shi, Z., Zhou, H., Surbiryala, J., Hu, Y., de Laat, C., Zhao, Z.: An automated customization and performance profiling framework for permissioned blockchains in a virtualized environment. In: 2019 IEEE International Conference on Cloud Computing Technology and Science (CloudCom). pp. 404-410. IEEE Computer Society (2019)

9. Sukhwani, H., Martínez, J.M., Chang, X., Trivedi, K.S., Rindos, A.: Performance modeling of pbft consensus process for permissioned blockchain network (hyperledger fabric). In: 2017 IEEE 36th Symposium on Reliable Distributed Systems (SRDS). pp. 253-255. IEEE (2017)

10. Taş, R., Tanrı̈över, Ö.Ö.: Building a decentralized application on the ethereum blockchain. In: 2019 3rd International Symposium on Multidisciplinary Studies and Innovative Technologies (ISMSIT). pp. 1-4. IEEE (2019)

11. Thakkar, P., Nathan, S., Viswanathan, B.: Performance benchmarking and optimizing hyperledger fabric blockchain platform. In: 2018 IEEE 26th International Symposium on Modeling, Analysis, and Simulation of Computer and Telecommunication Systems (MASCOTS). pp. 264-276. IEEE (2018)

12. Uriarte, R.B., Zhou, H., Kritikos, K., Shi, Z., Zhao, Z., De Nicola, R.: Distributed service-level agreement management with smart contracts and blockchain. Concurrency and Computation: Practice and Experience p. e5800 (2020)

13. Wüst, K., Gervais, A.: Do you need a blockchain? In: 2018 Crypto Valley Conference on Blockchain Technology (CVCBT). pp. 45-54. IEEE (2018)

14. Zhou, H., Hu, Y., Ouyang, X., Su, J., Koulouzis, S., de Laat, C., Zhao, Z.: Cloudsstorm: a framework for seamlessly programming and controlling virtual infrastructure functions during the devops lifecycle of cloud applications. Software: Practice and Experience 49(10), 1421-1447 (2019)

15. Zhou, H., Ouyang, X., Ren, Z., Su, J., de Laat, C., Zhao, Z.: A blockchain based witness model for trustworthy cloud service level agreement enforcement. In: IEEE INFOCOM 2019-IEEE Conference on Computer Communications. IEEE (2019) 